\title{
Nonperturbative tests of the renormalisation of mixed clover-staggered currents in lattice QCD
}

\author{
Bipasha Chakraborty* \\ SUPA, School of Physics and Astronomy, University of Glasgow, Glasgow G12 8QQ, UK \\ E-mail: b.chakraborty.1@research.gla.ac.uk
}

\section{Christine Davies}

SUPA, School of Physics and Astronomy, University of Glasgow, Glasgow G12 8QQ, UK

E-mail: Christine.Davies@glasgow.ac.uk

\section{Gordon Donald}

School of Mathematics, Trinity College, Dublin 2, Ireland

\section{Rachel Dowdall}

DAMTP, University of Cambridge, Wilberforce Road, Cambridge CB3 OWA, UK

\section{Jonna Koponen}

SUPA, School of Physics and Astronomy, University of Glasgow, Glasgow G12 8QQ, UK

\section{G. Peter Lepage}

Laboratory of Elementary Particle Physics, Cornell University, Ithaca, NY 14853, USA

\section{HPQCD Collaboration}

\begin{abstract}
The Fermilab Lattice and MILC collaborations have shown in one-loop lattice QCD perturbation theory that the renormalization constants of vector and axial-vector mixed clover-Asqtad currents are closely related to the product of those for clover-clover and asqtad-asqtad (local) vector currents. To be useful for future higher precision calculations this relationship must be valid beyond one-loop and very general. We test its validity nonperturbatively using clover and Highly Improved Staggered (HISQ) strange quarks, utilising the absolute normalication of the HISQ temporal axial current. We find that the renormalization of the mixed current differs from the square root of the product of the pure HISQ and pure clover currents by 2-3\%. We also compare discretization errors between the clover and HISQ formalisms.
\end{abstract}

31st International Symposium on Lattice Field Theory LATTICE 2013

July 29-August 3, 2013

Mainz, Germany

\footnotetext{
* Speaker.
} 


\section{Motivation}

The leptonic decay constants of the heavy-light mesons are important physical quantities in lattice QCD. They are needed to extract CKM matrix elements [1] from the measurements of the decay rate and thus need to be calculated precisely from lattice QCD to test the unitarity of the CKM matrix in the Standard Model as stringently as possible. A good test of lattice QCD is the comparison of results using different quark formalisms. Results for the $B$ and $B_{S}$ meson decay constants are summarized in Fig. 1 and the tension with experiment for $f_{B}$ shown [2]. The HPQCD collaboration have obtained separate results using NRQCD $b$ quarks [3] and HISQ $b$ quarks [4], both combined with HISQ light quarks, which are consistent within their $2 \%$ errors for $f_{B_{s}}$. The systematic uncertainties for the two methods are very different, with the NRQCD $b$ quark calculation having a significant contribution from the uncertainty in the current renormalization. There is no such uncertainty in the HISQ $b$ quark calculation but there the error is dominated by discretization errors. An alternative method from the Fermilab lattice/MILC collaborations uses heavy clover $b$ quarks. This method also gives a reasonably consistent result but with much larger errors coming from a number of sources. Here we test the method for determining the current renormalization in this formalism to see if the estimate of the error from that source is robust.

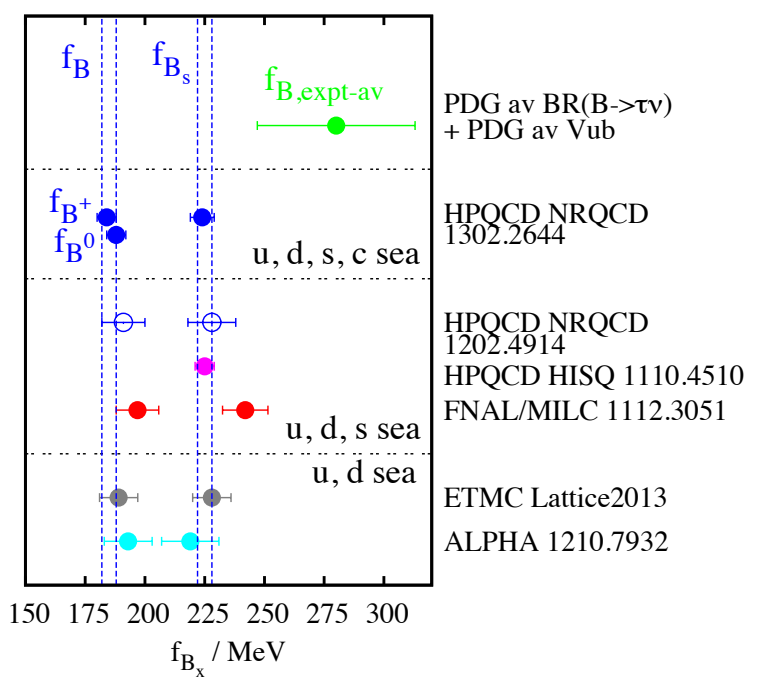

Figure 1: Results for the $B$ and $B_{s}$ meson decay constants using different formalisms and number of flavours of sea quarks.

\section{Our method}

The Fermilab/MILC collaboration used asqtad light valence quarks and clover $b$ and $c$ quarks for calculating the $\mathrm{B}$ and $\mathrm{D}$ meson decay constants [5]. In doing so, the heavy-light axial current renormalization constant $Z_{A_{Q q}^{\mu}}$ was calculated partly non-perturbatively and partly in one-loop perturbation theory. They proposed a definition relating the renormalization constants for the heavylight temporal axial current and the square roots of the renormalization constants for the heavy- 
heavy and light-light local temporal vector currents given by

$$
Z_{A_{Q q}^{4}}=\rho_{A_{Q q}^{4}} \sqrt{Z_{V_{q q}^{4}} Z_{V_{Q Q}^{4}}}
$$

It has been shown in one-loop perturbation theory that the correction factor $\rho_{A_{Q q}^{4}}$ has a small oneloop coefficient (see Fig. 2 from [6]). This result is used to argue that $\rho_{A_{Q q}^{4}}$ is close to unity for higher orders too, although this claim has not been explicitly demonstrated. We have carried out a completely non-perturbative calculation of the $\mathrm{Z}$ factors to test the validity of the Fermilab/MILC claim beyond one-loop. We have used HISQ-HISQ, clover-clover and mixed clover-HISQ currents made of strange valence quarks to see how close the $\rho_{A_{Q q}^{4}}$ is to unity. Since the claim must be general to be useful, it is not necessary for us to use asqtad quarks to test it. Instead we use HISQ quarks since they are further improved [7] and are being used in current state-of-the-art calculations [8].

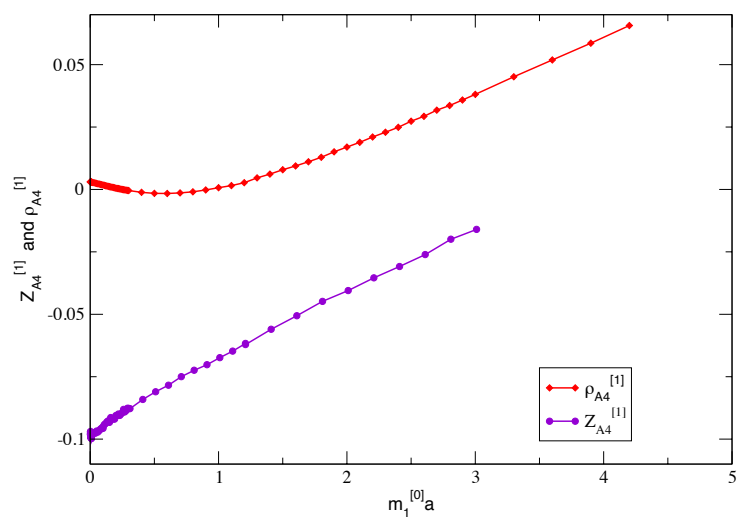

Figure 2: The one-loop coefficients of $\rho_{A_{Q q}^{4}}$ and $Z_{A_{Q q}^{4}}$ for the clover-asqtad case plotted against the bare clover quark mass [6].

We have chosen the strange quark as the valence quark as its mass too falls within the light quark mass region where (in fact, see Fig. 2) the claim for the smallness of the one-loop coefficient of $\rho_{Q q}$ holds [6]. The valence strange quark mass was tuned to give the mass of a fictitious pseudoscalar meson known as $\eta_{s}$ to be $688.5 \mathrm{MeV}$ [9]. We made the $\eta_{s}$ from two HISQ strange quarks, two clover strange quarks and mixing one HISQ and one clover strange quark.

The decay constant of the $\eta_{s}$ is related to the matrix element of the temporal axial current between the $\eta_{s}$ and the vacuum and is given by $\left\langle 0\left|A^{4}\right| \eta_{s}(0)\right\rangle=m_{\eta_{s}} f_{\eta_{s}}$ when the $\eta_{s}$ is at rest. We have utilized the fact that the HISQ-HISQ temporal axial current is absolutely normalized and via the PCAC relation we can then obtain $f_{\eta_{s}}$ with no normalization factor. We then obtained the temporal axial vector current renormalization for the clover-HISQ discretization using the relation $Z_{A_{C l-H}^{4}} f_{\eta_{s}}^{C l-H}=f_{\eta_{s}}^{H-H}$.

The renormalization factors for the clover-clover and HISQ-HISQ local vector currents are obtained by demanding at zero momentum transfer that $1=Z_{V_{q q}^{4}}\left\langle H_{q}\left|V_{q q}^{4}\right| H_{q}\right\rangle$. The matrix element is extracted by fitting the two-point and three-point correlators simultaneously. A point to note: We need to use an unstaggered (clover in our case) quark propagator as the spectator quark in the three point correlators for the HISQ-HISQ local vector current since a purely staggered three-point function with the same mesons at either end would vanish in this case (because the local vector current is not a taste-singlet). 
For our calculation, we have used three lattice ensembles with lattice spacings $a \approx 0.15 \mathrm{fm}$ (very coarse), $0.12 \mathrm{fm}$ (coarse) and $0.09 \mathrm{fm}$ (fine). The ensembles were generated by MILC using the light, strange and charm HISQ quarks in the sea where $m_{l} / m_{s} \approx 0.2$ [10]. The valence HISQ strange quark mass was tuned using the Wilson flow parameter $w_{0}$ to set the lattice spacing [9] and listed in table 1 . There we have also listed tuned valence clover $\kappa_{s}$ on each ensemble, the number of configurations used $n_{c f g}$ and the number of time sources per configuration $n_{t}$.

Table 1: List of simulation parameters

\begin{tabular}{cccccc}
\hline \hline Set & $a(\mathrm{fm})$ & $a m_{s}{ }^{H I S Q, v a l}$ & $\kappa_{s}^{\text {Clover,val }}$ & $n_{c f g}$ & $n_{t}$ \\
\hline Very Coarse & $0.1543(8)$ & 0.0705 & 0.14082 & 1021 & 12 \\
\hline Coarse & $0.1241(7)$ & 0.0541 & 0.13990 & 527 & 16 \\
\hline Fine & $0.0907(5)$ & 0.0376 & 0.13862 & 504 & 16 \\
\hline \hline
\end{tabular}

\section{Our Results}

Table 2 summarizes our results for testing the relation $Z_{A^{4} C l-H}=\rho_{A^{4}{ }_{C l-H}} \sqrt{Z_{V^{4}{ }_{C l-C l}} Z_{V^{4} H-H}}$.

Table 2: Our results for $Z_{A^{4}}, Z_{V^{4}}$ and $\rho_{A^{4}{ }_{a b}}$

\begin{tabular}{ccccc}
\hline \hline Set & Combinations & $Z_{A^{4}}$ & $Z_{V^{4}}$ & $\rho_{A^{4}}{ }_{a b}$ \\
\hline VC & $\mathrm{H}-\mathrm{H}$ & 1.000 & $0.9887(20)$ & - \\
& $\mathrm{Cl}-\mathrm{Cl}$ & $0.2046(4)$ & $0.2045(3)$ & - \\
& $\mathrm{Cl}-\mathrm{H}$ & $0.4642(6)$ & - & $1.0322(21)$ \\
\hline $\mathrm{C}$ & $\mathrm{H}-\mathrm{H}$ & 1.000 & $0.9938(17)$ & - \\
& $\mathrm{Cl}-\mathrm{Cl}$ & $0.2096(4)$ & $0.2071(4)$ & - \\
& $\mathrm{Cl}-\mathrm{H}$ & $0.4656(4)$ & - & $1.0263(36)$ \\
\hline $\mathrm{F}$ & $\mathrm{H}-\mathrm{H}$ & 1.000 & $0.9944(10)$ & - \\
& $\mathrm{Cl}-\mathrm{Cl}$ & $0.2152(4)$ & $0.2116(4)$ & - \\
& $\mathrm{Cl}-\mathrm{H}$ & $0.4679(7)$ & - & $1.0199(33)$ \\
\hline \hline
\end{tabular}

After calculating the $\mathrm{Z}$ factors completely nonperturbatively we find $\rho_{A^{4} C l-H}$ is indeed close to 1.0 up to all orders of lattice perturbation theory with a maximum deviation of $\sim 3 \%$ on very coarse lattice. Our results for $\rho_{A_{C l-H}^{4}}$ are shown and compared to the Fermilab/MILC results for cloverasqtad using their one-loop perturbative coefficients and two different values of the clover quark mass in Fig. 3. The equivalent perturbative results for clover-HISQ could be different because asqtad and HISQ are different formalisms. However, the fact that our numbers do lie further from 1 than the Fermilab/MILC results suggests that it may be sensible to increase the perturbative errors in their results to $2 \%$. This would then allow for the possibility that the clover-asqtad all-orders result is as far from 1 as our clover-HISQ result.

We also note from the table 2 that $Z_{V^{4} C l-C l}$ and $Z_{A^{4} C l-C l}$ are very close to each other, despite the fact that clover quarks break chiral symmetry. 


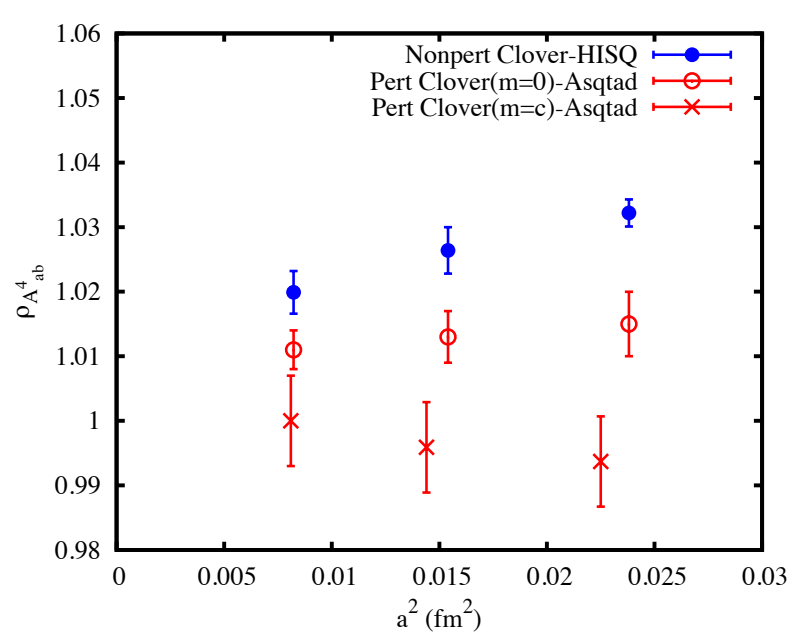

Figure 3: Our results for $\rho_{A^{4}}{ }_{a b}$ (dots), plotted against the square of the lattice spacing and compared to the one-loop results from Fermilab/MILC for mixed clover-asqtad currents with clover charm (crosses) and clover light (open circles) quarks.

\section{Discretization effects}

We also discuss the discretization effects coming from the different methods used in our present calculation. The difference of the $\eta_{s}$ mass obtained from the HISQ-HISQ and clover-HISQ correlators (when both HISQ and clover quark masses are tuned to that of the strange quark) is plotted in Fig. 4 and is clearly a discretization effect, vanishing quadratically as the lattice spacing goes to zero.

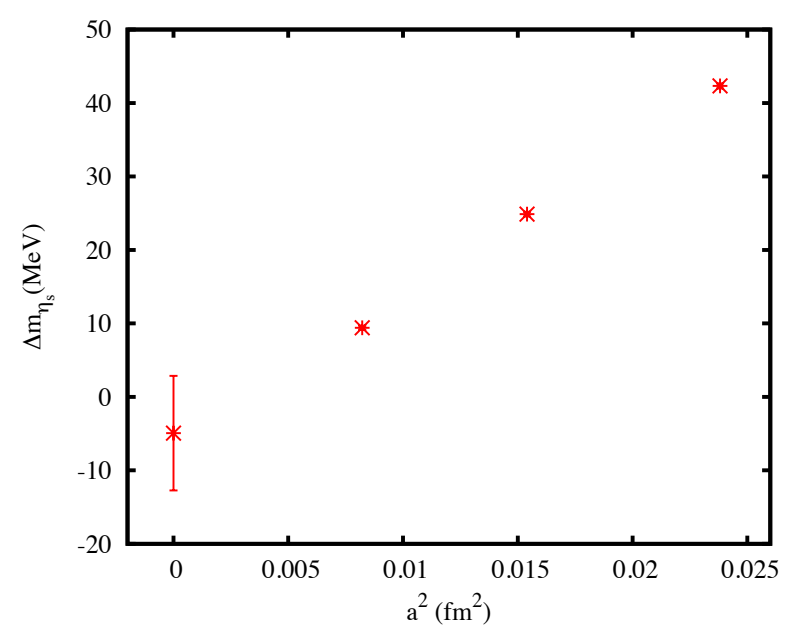

Figure 4: The difference of the $\eta_{s}$ mass obtained from the HISQ-HISQ and clover-HISQ methods

For another comparison of discretization effects we calculate the mass of the vector meson $\phi$ and then extrapolate $m_{\phi}-m_{\eta_{s}}$ to the continuum $a=0$. From the extrapolation plot in Fig. 5 we see all three methods of calculating correlators agree in the continuum limit as expected. The HISQ-HISQ discretization errors are much smaller than the clover-clover and the clover-HISQ 
discretizations. The accurate HISQ-HISQ results show a value in continuum limit which is higher than experiment. The $\phi$ is not a gold-plated meson, having a strong decay to $K \bar{K}$ and further study is needed to uncover what the impact of this decay channel is.
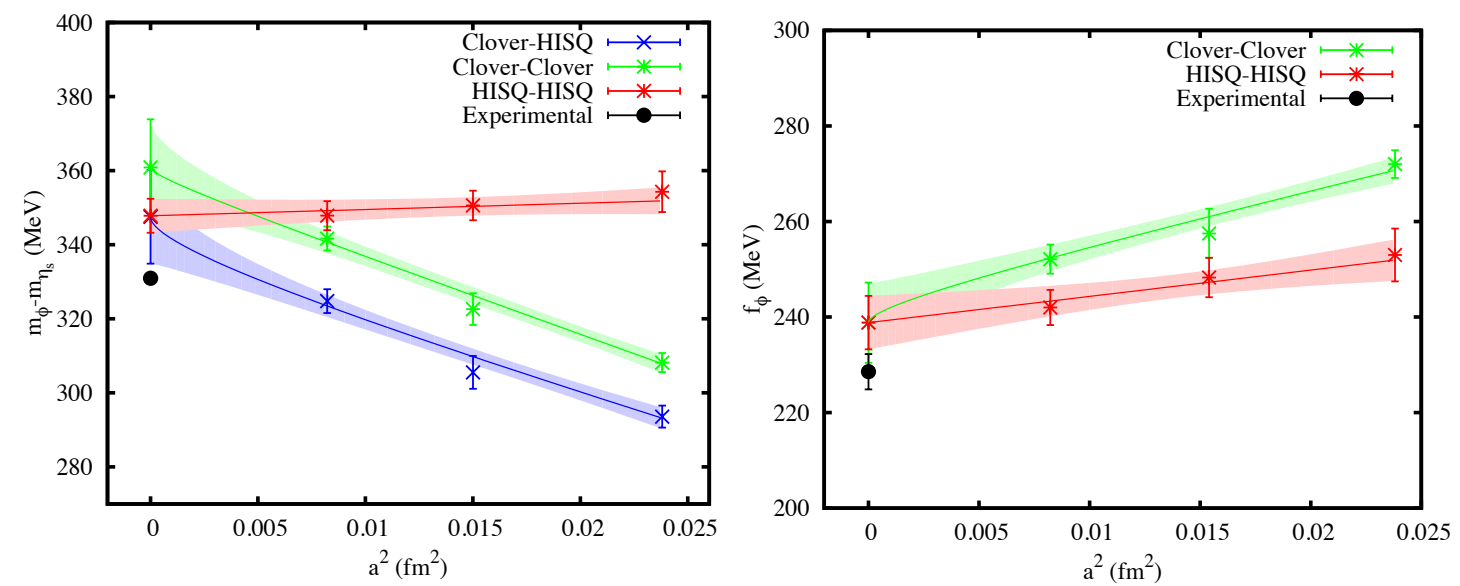

Figure 5: On the left: $m_{\phi}-m_{\eta_{s}}$ calculated with different quark formalisms and extrapolated to $a=0$. On the right $f_{\phi}$ calculated with different quark formalisms and extrapolated to $a=0$.

We also calculate the $\phi$ decay contant using the renomalization constants $Z_{V^{4} \mathrm{Cl}-\mathrm{Cl}}$ and $Z_{V^{4} H-H}$ determined earlier. This is also plotted as a function of lattice spacing in Fig. 5 with extrapolation to the continuum. As before, we find that all discretizations agree in the continuum and again the HISQ-HISQ case shows the smallest discretization errors. In this case, the lattice $f_{\phi}$ results match with the experimental result obtained from $\Gamma\left(\phi \rightarrow e^{+} e^{-}\right)$[2] within $1.5 \sigma$.

\section{Conclusion and Ongoing Work}

The following conclusions are drawn based on our present work:

- $\rho_{A^{4} C l-H}$ is close to 1.0 with a maximum $\sim 3 \%$ deviation using the nonperturbative lattice calculations with pure HISQ, pure clover and mixed clover-HISQ currents.

- Based on our numbers we would recommend increasing the errors in the Fermilab/MILC results from $0.7 \%$ to $2.0 \%$ in one loop perturbative calculation of $\rho_{A^{4} C l-H}$.

- Discretization errors are significantly smaller for HISQ than clover.

We are also studying $\rho_{V^{4} \mathrm{Cl}-H}$ using $Z_{V^{4} \mathrm{Cl}-\mathrm{H}}$. We will extend our study of the vector meson $\phi$ to the MILC gluon field configurations that include physical $\mathrm{u} / \mathrm{d}$ sea quarks to test more carefully the effect of it not being gold-plated.

\section{Acknowledgements}

We are grateful to the MILC collaboration for the use of their configurations and to A. Kronfeld for useful discussions. Computing was done on the Darwin supercomputer at the University of Cambridge as part of STFC's DiRAC facility. We are grateful to the Darwin support staff for 
assistance. Funding for this work came from the NSF, the Royal Society, the Wolfson Foundation and STFC.

\section{References}

[1] C. Davies, PoS LATTICE2011, 019 (2011), 1203.3862.

[2] Particle Data Group, J. Beringer et al., Phys. Rev. D 86, 010001 (2012).

[3] HPQCD Collaboration, R. Dowdall et al., (2013), 1309.4610.

[4] C. McNeile, C. Davies, E. Follana, K. Hornbostel, and G. Lepage, Phys.Rev. D85, 031503 (2012), 1110.4510 .

[5] Fermilab Lattice and MILC Collaborations, A. Bazavov et al., Phys. Rev. D 85, 114506 (2012).

[6] A. X. El-Khadra, E. Gamiz, A. S. Kronfeld, and M. A. Nobes, PoS LAT2007, 242 (2007), 0710.1437.

[7] HPQCD Collaboration, E. Follana et al., Phys. Rev. D 75, 054502 (2007).

[8] HPQCD Collaboration, R. Dowdall, C. Davies, R. Horgan, C. Monahan, and J. Shigemitsu, Phys.Rev.Lett. 110, 222003 (2013), 1302.2644.

[9] HPQCD Collaboration, R. Dowdall, C. Davies, G. Lepage, and C. McNeile, Phys.Rev. D88, 074504 (2013), 1303.1670.

[10] MILC Collaboration, A. Bazavov et al., Phys. Rev. D 87, 054505 (2013). 